\title{
Impact of Pilot Delay and Non-Responsiveness on the Safety Performance of Airborne Separation
}

\author{
Maria Consiglio, ${ }^{1}$ Sherwood Hoadley, ${ }^{2}$ David Wing, ${ }^{3}$ Brian Baxley ${ }^{4}$ and Danette Allen ${ }^{5}$ \\ NASA Langley Research Center, Hampton, VA, 23681
}

\begin{abstract}
Assessing the safety effects of prediction errors and uncertainty on automationsupported functions in the Next Generation Air Transportation System concept of operations is of foremost importance, particularly safety critical functions such as separation that involve human decision-making. Both ground-based and airborne, the automation of separation functions must be designed to account for, and mitigate the impact of, information uncertainty and varying human response. This paper describes an experiment that addresses the potential impact of operator delay when interacting with separation support systems. In this study, we evaluated an airborne separation capability operated by a simulated pilot. The experimental runs are part of the Safety Performance of Airborne Separation (SPAS) experiment suite that examines the safety implications of prediction errors and system uncertainties on airborne separation assistance systems. Pilot actions required by the airborne separation automation to resolve traffic conflicts were delayed within a wide range, varying from five to 240 seconds while a percentage of randomly selected pilots were programmed to completely miss the conflict alerts and therefore take no action. Results indicate that the strategic ASAS functions exercised in the experiment can sustain pilot response delays of up to $\mathbf{9 0}$ seconds and more, depending on the traffic density. However, when pilots or operators fail to respond to conflict alerts the safety effects are substantial, particularly at higher traffic densities.
\end{abstract}

\section{Nomenclature}

ADS-B = Automatic Dependence Surveillance-Broadcast

$\mathrm{AOP}=$ Autonomous Operations Planner

ARTCC = Air Route Traffic Control Center

ASAS $=$ Airborne Separation Assistance System

ASTOR $=$ Aircraft Simulation for Traffic Operations Research

ATOL $=$ Air Traffic Operations Laboratory

ATOS $=$ Airspace $\&$ Traffic Operations Simulation

$\mathrm{CD} \quad=$ Conflict Detection

$\mathrm{CR} \quad=$ Conflict Resolution

FLOS $=$ First Loss of Separation

LOS $=$ Loss of Separation

NAS $\quad=$ National Airspace System

NextGen $=$ Next Generation Air Transportation System

$\mathrm{nmi} \quad=$ Nautical Miles

PM $=$ Pilot Model

SPAS $=$ Safety Performance of Airborne Separation

\footnotetext{
${ }^{1}$ Research Engineer, Crew Systems and Aviation Operations Branch, NASA LaRC/MS 152, AIAA Member.

${ }^{2}$ Research Engineer, Crew Systems and Aviation Operations Branch, NASA LaRC/MS 152, past AIAA Associate Fellow.

${ }^{3}$ Research Engineer, Crew Systems and Aviation Operations Branch, NASA LaRC/MS 152, AIAA Member.

${ }^{4}$ Research Engineer, Crew Systems and Aviation Operations Branch, NASA LaRC/MS 152, AIAA Member.

${ }^{5}$ Research Engineer, Crew Systems and Aviation Operations Branch, NASA LaRC/MS 152, AIAA Member.
} 


\section{Introduction}

A SSESSING the safety effects of human response characteristics on automation-supported functions in the Next Generation Air Transportation System (NextGen) concept of operations ${ }^{1}$ is of foremost importance, particularly safety critical functions, such as separation, that involve human decision-making. The automation of separation functions, both ground-based and airborne, must be designed to account for, and mitigate the impact of, varying human response. Delayed responses to conflict alerts may result in late resolutions possibly leading to losses of separation. Automation systems for separation require operators to interact in a timely manner responding to predicted hazardous conditions in accordance with procedures and training. This paper describes an experiment that addresses the potential impact of operator delay when interacting with separation support systems within the context of the aircraft crew interacting with an Airborne Separation Assistance Systems (ASAS). By understanding the impact of delayed pilot responses to conflict alerts we can establish design guidelines for crew procedures and automation alert timing in ASAS applications. Similar studies need to be performed for all automation-enabled separation assurance applications that rely on the human operators to preserve safe separation.

This study is part of the Safety Performance of Airborne Separation (SPAS) experiment ${ }^{2}$ suite that comprises a series of batch simulation studies investigating the safety impact of prediction errors and system uncertainties on ASAS applications. Current SPAS experiments are based on scenarios consisting of randomized routes in a generic high-density airspace in which all aircraft are constrained to the same flight level. Sustained average traffic density is varied from 11.2 to 21.4 aircraft per 10,000 square $\mathrm{nmi}$, approximating up to about 10 times today's traffic density in a typical sector. During the progression of experiments, various errors, uncertainties, delays, and other variables potentially impacting system safety will be incrementally introduced to analyze the effect on safety of the individual factors as well as their interaction and collective effect. Results from the baseline SPAS study ${ }^{2}$ indicate that at five times the typical traffic density of today's National Airspace System (NAS) and under the assumptions of the study, airborne separation can be safely performed.

In the current study, pilot actions required by the ASAS automation to resolve traffic conflicts were varied over a wide range of response times, varying from 5 to 240 seconds, while a percentage of randomly selected pilots were programmed to completely miss the conflict alerts and therefore take no action.

The paper is organized as follows: Section II presents a brief summary of previous work, Section III describes the simulation platform, and Sections IV and V describe the experiment design and results. Finally, Section VI presents conclusions and future direction research.

\section{Background}

The addition of automation to support the separation function is a response to the capacity limiting effect of human-based separation control of today's system ${ }^{3,4}$. Automation can remove workload bottlenecks, improve prediction of conflicts as traffic patterns become more dense and complex, and provide conflict-free maneuvers alternatives to human operators. Integrated air/ground operational concepts have been proposed in which some aircraft crews exercise separation functions aided by ASAS tools on the flight deck, while air traffic controllers exercise ground based separation control for non-ASAS-equipped aircraft and terminal operations ${ }^{5}$. ASAS systems integrate advanced decision-aiding automation into aircraft avionics. These decision aids rely on data-linked, broadcast surveillance information that includes aircraft velocity vectors and limited flight plan information through a surveillance capability such as the Automatic Dependent Surveillance - Broadcast (ADS-B) ${ }^{6}$. The automation is designed to detect conflicts between aircraft and generate conflict resolution routes and conflict-free maneuvering advisories ${ }^{7,8,9}$.

Until now, safety evaluation of SA applications has, for the most part, been based on studies using simulation tools that seldom include models of system uncertainties ${ }^{10,11,12}$ and often make many simplifying assumptions such as perfect navigation performance and absence of prediction errors or off-nominal conditions. Analytical and numerical approaches to the analysis of safety using uncertainty propagation models often require many simplifying assumptions to keep the size of the models manageable or computation time within reasonable limits ${ }^{13}$. Monte Carlo simulations are frequently an alternative when the analysis requires higher fidelity, although simplifying assumptions are often required to more quickly achieve large amounts of data. However, statistically valid predictions are elusive when based on the occurrence of events that are seldom observed such as the critical separation violations in normal air traffic conditions. For that reason, special techniques for the modeling of rare event probabilities are being explored in the context of Air Traffic Management (ATM) operations ${ }^{14}$. Although these studies are key to understanding the behavior of large complex systems such as the NextGen, it is important to 
complement them with high fidelity, detailed studies that incorporate models of uncertainty sources, measure performance in the presence of prediction errors and operator performance variability.

The SPAS simulation study attempts to characterize and quantify the safety performance of ASAS applications using a simulation platform that includes high fidelity models of aircraft dynamics, flight management system, data link communications, and conflict detection and resolution functions as described in the next section. The baseline set of runs for the SPAS experiment, completed in the spring of 2007, included scenarios with no system uncertainties or prediction errors ${ }^{2}$. Results from that study indicated that within the experimental conditions and assumptions, safety was preserved with no losses of separation observed for traffic densities much higher than current levels. For the baseline SPAS test runs described in the aforementioned study ${ }^{2}$, ADS-B reception was perfect (i.e., all messages received at all ranges), full aircraft trajectory intent was shared, the pilot responded correctly to all traffic alerts with no delays, and wind predictions were accurate. Results showed that at five times the typical traffic density of today's NAS, utilizing only airborne strategic conflict detection and resolution logic with a 10-minute look-ahead time, safe separation of aircraft can be maintained under the assumptions and conditions of the test. Subsequent studies underway and planned seek to identify the conditions and estimate the traffic levels that could compromise safety in the presence of various sources of uncertainty.

One of the problems in conducting a pilot response delay study on airborne separation is the lack of prior experimental or measured data describing valid pilot response delay ranges in similar circumstances or for similar SA systems. These applications are context sensitive and human performance is influenced by specific training and procedures as well as other required cockpit tasks. As indicated in a report on testing and designing new cockpit alerting systems ${ }^{15}$, there are new applications and procedures requiring alerting systems that "self-activate in timecritical, hazardous situations". Multiple factors can affect pilot response time and pilot conformance, such as lack of trust as a result of frequent occurrences of false alerts or conflicting training instructions. The operational procedure within which the alerting system is used is likely to affect the operator's response time. If pilots are trained and expected to react within a certain time period and are given responsibility for separation assurance, the response time would be required to be within a bounded time range. In some cases, the alerting system is part of a procedure that requires pilot compliance with immediate actions within a given time, such as the TCAS II procedure in which pilots are expected to act within 5 seconds of an alert. While there is a lack of studies and operational experience on which to base the requirements for pilot response times for airborne separation it is possible to investigate the effects of delayed pilot responses on safety using ad-hoc time delay ranges to measure the performance of relevant safety metrics. For this study, pilot response time ranges were configured to reach levels that could compromise separation in order to observe potential safety degradation. Additional experiment outcomes include procedure design guidelines for required pilot response times for future systems.

\section{The Simulation Platform}

\section{A. The Airspace \& Traffic Operations Simulation}

The SPAS experiments are being conducted at the Air Traffic Operations Laboratory (ATOL) at NASA Langley Research Center utilizing a distributed simulation platform that includes a network of aircraft simulators interconnected through a High Level Architecture (HLA) communication infrastructure. The simulation platform, known as the Airspace \& Traffic Operations Simulation $(\mathrm{ATOS})^{16}$ can be used for batch Monte Carlo studies as well as real-time human-in the-loop experiments. The ATOS is comprised of hundreds of real-time, high fidelity aircraft simulators equipped with experimental displays and pilot interfaces.

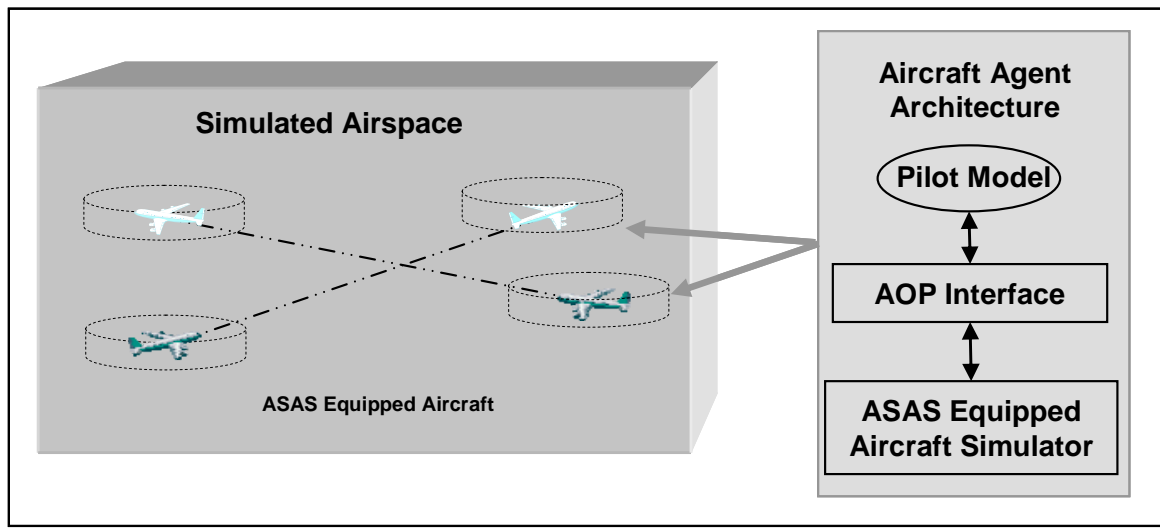

Figure 1: SPAS Simulation Platform

Each aircraft simulator is referred to as the Aircraft Simulation for Traffic Operations Research (ASTOR). 
Conceptually, the batch simulation platform is a multi-agent system composed of multiple distributed autonomous software agents modeling ASAS equipped aircraft flown by a rule-based pilot model as shown in Fig. 1. Each aircraft agent has its own view of the airspace, and there is no centralized system controlling or modifying the paths of the aircraft or behavior of the pilot models.

Each ASTOR includes a six degree-of-freedom dynamic airplane model, a Flight Management System (FMS), Mode-S ADS-B datalink capability, and a prototype ASAS called the Autonomous Operations Planner (AOP) that is described in the next section. For batch simulations, all ASTORS are "flown" by a rule-based pilot model (PM) that performs the basic pilot actions for airborne conflict management as described below.

\section{B. Airborne Conflict Management System}

The airborne conflict management system used in this experiment series is the Autonomous Operations Planner (AOP), a NASA-developed research model of an airborne automation system built for the study of advanced distributed air-ground operational concepts ${ }^{7}$. The AOP provides an integrated suite of capabilities for managing traffic conflicts and trajectory changes from the flight deck perspective, including conflict detection, resolution, prevention, and trajectory constraint conformance. These capabilities are developed to a level of fidelity sufficient for research of complete airborne responsibility for self-separation as defined by the Principles of Operation of ASAS Category 4, "Airborne Self-Separation"17. In the JPDO NextGen Concept of Operations, they correspond to the functions of Separation Management and aspects of Trajectory Management ${ }^{1}$.

The intent-based conflict detection (CD) function of AOP uses state and intent data received from other traffic aircraft over ADS-B in combination with ownship state data, auto-flight mode settings, and flight plan information to deterministically predict future losses of separation. AOP also has a second, independent, CD system that uses state-vector projections to detect flight crew blunders and prediction faults of the intent-based CD system and other short time horizon conflicts. Conflict alerting is modeled after the multi-alert-level approach recommended by RTCA $^{18}$. The timing of alerts between aircraft are staggered as a method for incorporating a right-of-way rule set (i.e., priority rules), based roughly on the set used in Visual Flight Rules ${ }^{19}$. In the current study, the simple computer-based pilot model reacted to alerts within a normally distributed timing scheme defined by the parameters of the experiment, described later. When alerted to a conflict, the pilot model's action was to request a fully-conflictfree resolution trajectory from AOP.

For conflict resolution (CR), AOP contains both strategic and tactical capabilities. Tactical CR refers to openloop vectors or altitude changes to solve conflicts with no predetermined reconnection plan. A Strategic CR refers to the single action of modifying the flight plan such that the conflict is solved and the aircraft reconnects to the previous trajectory. In generating solutions, AOP's strategic CR system takes into account all known trajectory constraints, including trajectories of nearby traffic, airspace hazards, ownship performance limits, and required time of arrival (RTA) constraints. A genetic algorithm is employed to search within a set of pre-defined geometric patterns to generate CR trajectories that simultaneously accommodate these constraints ${ }^{20}$. Once a suitable CR trajectory is calculated, and it is verified conflict free for a nominal time of 20 minutes based on current traffic data, it is presented to the flight crew for review. Immediately upon execution of the route, ADS-B broadcasts the new ownship intent as a series of trajectory change points.

AOP also includes functions for conflict prevention, including at-a-glance maneuver restriction symbology for the flight crew and support for tactical / strategic trajectory probing (also known as provisional CD). These functions were not required for this study and were therefore disabled.

\section{The Pilot Model}

Pilot model agents are self-contained agents capable of making independent decisions, and taking actions based upon their perceived state of the traffic and airspace. The PM reactive rulesbased logic was designed to interact with the conflict management system by responding to conflict alerts with the action of accepting a conflict resolution advisory.

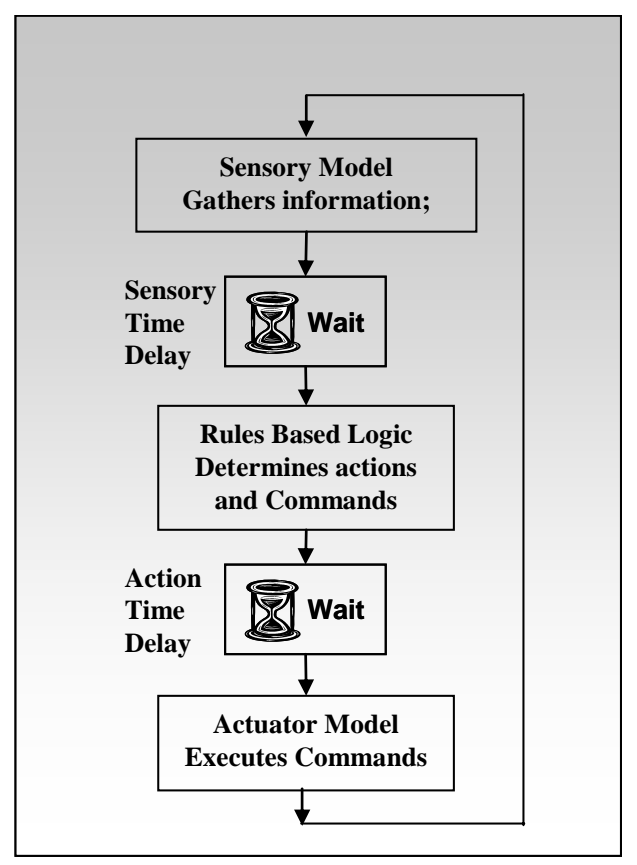

Figure 2: Pilot Model Architecture 
The PM design is composed of three parts: a sensory input model, a rule-based decision model, and an actuator response model as shown in Fig. 2. The sensory input model gathers information by detecting changes in the AOP interface such as a new conflict alert. A sensory time delay was implemented to model cases when a pilot does not notice a conflict alert immediately. This delay can be individually configured for each action that the PM is designed to perform. For the strategic conflict detection and resolution interface that is exercised in this experiment, there are three different pilot actions required and they are described in more detail in Section IV. The rules module determines the appropriate action and commands the actuator module to execute it. The actuator model simulates the pilot action required to execute a command, such as pushing a button, and its responses are also governed by a time delay algorithm.

\section{Experiment Description}

\section{A. Experiment Scenarios and Assumptions}

A simulated en route airspace modeled for the SPAS experiment is depicted in Fig. 3. The test region, a notional airspace en route sector, is modeled as a circular area with a diameter of $160 \mathrm{nmi}$. The test region is surrounded by an initialization region, the outer boundary of which is the location where aircraft are initialized in the simulation. This initialization method provides each aircraft's AOP with a full 10minute look-ahead time for detecting conflicts that occur within the test region. Aircraft are generated at random points on the outer circle initially with straight trajectories that traverse the test

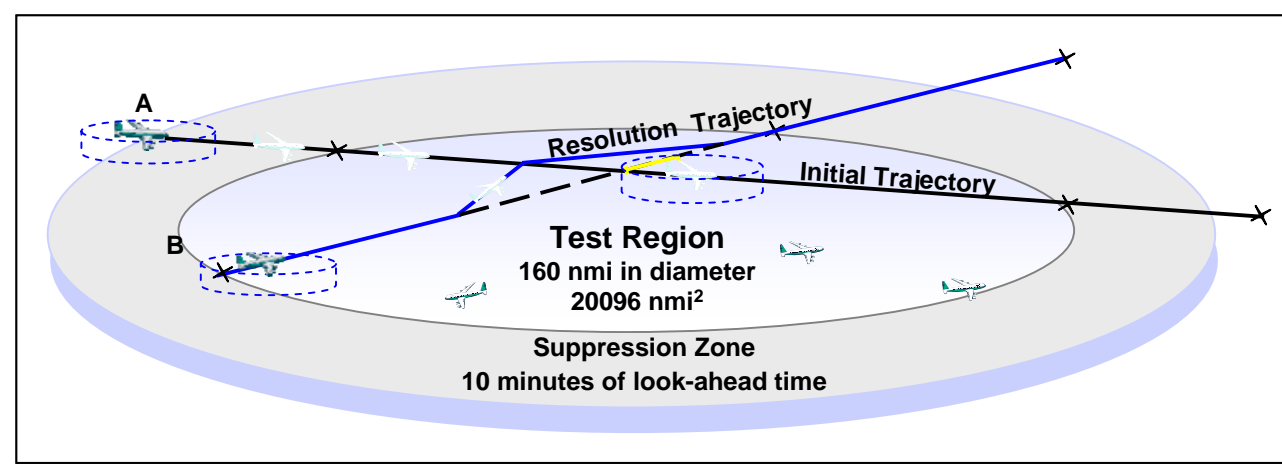

Figure 3. SPAS Scenario Design region at random angles and continue to a waypoint with a required time of arrival. All aircraft in the simulation are ASAS equipped and fly at the same altitude so as to constrain the scenarios to lateral conflict resolutions only and to achieve higher traffic densities. More details on the SPAS scenario design can be found in the Preliminary SPAS experiment report $^{2}$.

For this study, the auto-flight system remained coupled to the FMS for lateral navigation such that there was no tactical maneuvering. No altitude changes were permitted. There were no wind prediction errors or ADS-B message degradation due to signal range or interference. The AOP was configured to exercise only the intent-based CD and strategic CR capabilities with a look-ahead time of 10 minutes and a separation minimum of 5.1 nautical miles. ADS-B communications included full intent data. A priority rule (i.e., right of way) system was in effect that prevented undesirable synchronicity of resolution maneuvers by both aircraft involved in a conflict. Aircraft given priority for a given conflict had their alerts delayed 3 minutes while the other conflicting aircraft were alerted immediately. In Fig. 3 aircraft B has an initial trajectory that is in conflict with aircraft A, just entering the initialization zone. Aircraft B resolves the conflict by modifying its fly path as shown in the diagram.

The state-based CD and the tactical CR capability were disabled to allow the current study to focus on strategic conflict management. The strategic resolution logic attempts to find an RTA-conforming route that is conflict-free for 20 minutes. Nominal look-ahead time is 10 minutes, but the strategic CR will attempt resolutions with as little as 2 minutes to separation loss. If a resolution is found, it is guaranteed to preserve separation in the absence of prediction errors, even if only one aircraft executes it. If a strategic resolution cannot be found in time, the system would normally transition to a tactical resolution phase (typically with three minutes to go), which is not present in this experiment. The result of not having the tactical back-up system is that conflicts irresolvable by the strategic system of both aircraft will result in separation loss. Nevertheless, all losses of separation (LOS) must be accounted for regardless of their severity. Future studies will incorporate both the strategic and tactical capabilities. 


\section{B. The Pilot Model Configuration}

For this experiment the PM was designed to interact with the human interface currently implemented for the ASTOR's strategic conflict detection and resolution functionality. This interface requires three pilot actions: First, when a conflict is detected and its alert is shown on the navigation display, the PM must request AOP to compute a resolution trajectory. This action represents the pilot's first awareness of a potential conflict and it is modeled by a delay shown in Fig. 4 as d1. The PM reaction to the conflict alert is to request AOP to compute a resolution route. Once computed, a resolution route is shown to the pilot on the experimental navigation display.

The time elapsed between the PM request and when the resolution route is completed is a dependent metric referred to as the Resolution Cycle Time. This is the time it takes AOP to compute a conflict free trajectory based on all the known traffic information. This time is strongly affected by traffic density and in this

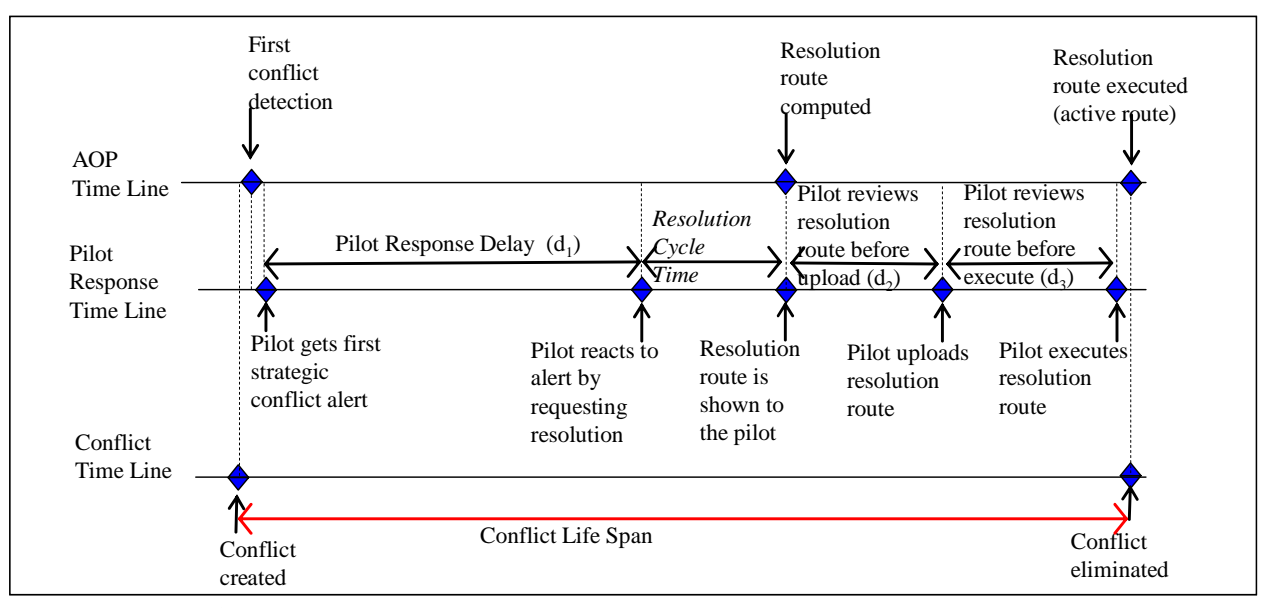

Figure 4. Conflict Alert Timeline current study ranged between mean values of 5 to 25 seconds for traffic densities ranging from 11.4 to 20.3 average aircraft per $10000 \mathrm{nmi} 2$. Second, when the new route is displayed, a pilot would have the opportunity to review the new trajectory before uploading it. This is modeled by the PM as the d2 delay shown in Fig. 4 . When the resolution route is uploaded, it becomes a MOD route for the FMS and the pilot is required to execute it before it becomes the active route. The time before this last PM action is modeled with a time delay shown as d3 in Fig. 4. The traffic conflict is then removed from the display. All PM delays for this experiment were modeled as normally distributed random variables with mean values ranging from 3.5 to 240 seconds. The lowest delay value is used to represent a "typical" pilot response delay. The responsiveness factor represents pilots that ignore conflicts alerts and do not attempt to resolve them. The responsiveness factor is modeled as a uniformly distributed random variable representing the percentage of times that all pilots ignore conflicts. This variable is sampled for each individual conflict, therefore, it is possible for two pilots involved in a conflict to both ignore it and never request a resolution. In the current study, the pilot model was programmed to execute the first available CR trajectory with an average delay of five seconds from the time the resolution route is shown.

\section{Experiment Design}

A three-factor, response surface experiment design was used to explore the effect of average traffic density, pilot response delay and pilot non-responsiveness, on safety. The two safety metrics of interest in this experiment are losses of separation (LOS) and the time remaining before LOS at resolution execution. The experiment consisted of 85 simulation runs in which the three factors were assigned values in the Table 1: Experiment Design

\begin{tabular}{|l|l|l|l|}
\hline Factors & Units & Min & Max \\
\hline Traffic Density & $\begin{array}{l}\text { Number of Aircraft per } \\
10000 \mathrm{nmi}^{2}\end{array}$ & 11.2 & 21.4 \\
\hline Pilot Delay & Seconds & 3.5 & 240 \\
\hline $\begin{array}{l}\text { Pilot Non- } \\
\text { Responsiveness }\end{array}$ & $\begin{array}{l}\text { Percentage of pilots not } \\
\text { responding to conflict } \\
\text { alerts }\end{array}$ & 0 & 10 \\
\hline
\end{tabular}
ranges shown in Table 1.

Table 2 shows the relationship of mean traffic density levels tested to current-day levels, as well as the number of flight hours of data collected and conflicts recorded. In total, 6978.5 flight hours of data were collected, and 
24384 conflicts were generated. As expected, conflicts per flight hour increased with traffic density. It should be noted that this metric reflects all traffic in the airspace, not conflicts per single aircraft flight hour. The approximate mean and peak ratios are based on an analysis of traffic demand levels in the NAS that estimated the traffic count for every high altitude sector in the United States, at each flight level, for the 24 hour period of 19 February 2004 (ref. SPAS baseline). From this Table 2: Summary of Data Collection Runs

\begin{tabular}{|c|c|c|c|c|}
\hline $\begin{array}{c}\text { Average } \\
\text { Density } \\
\text { Per } \mathbf{1 0 0 0 0} \\
\text { nmi }^{2}\end{array}$ & $\begin{array}{c}\text { Approximate } \\
\text { Traffic Ratio } \\
\text { (mean/peak) }\end{array}$ & $\begin{array}{c}\text { Flight } \\
\text { Hours }\end{array}$ & $\begin{array}{c}\text { Total } \\
\text { Conflicts }\end{array}$ & $\begin{array}{c}\text { Conflicts } I \\
\text { Flight } \\
\text { Hour }\end{array}$ \\
\hline 11.2 & $5 \mathrm{X} / 3 \mathrm{X}$ & 1375.7 & 3273 & 2.38 \\
\hline 16.3 & $8 \mathrm{X} / 6 \mathrm{X}$ & 1918.4 & 6361 & 3.32 \\
\hline 21.4 & $12 \mathrm{X} / 8 \mathrm{X}$ & 3684.4 & 14750 & 4.00 \\
\hline
\end{tabular}
data, the mean and maximum traffic count for FL310 (the busiest altitude within the selected sector) was determined for the median-density sector ZOA31. The traffic levels were then normalized to produce densities based on $10,000 \mathrm{nmi}^{2}$. The ranges used in this study represent an estimated five to twelve times the average traffic density of the median sector and three to eight times its peak density.

\section{Experiment Results}

\section{A. Impact of Traffic Density}

The effect of average traffic density with normal pilot delays and all pilots responding can be observed in Table 3. Preliminary analysis indicates that at the highest density level and under the constrained conditions of the experiment scenarios, the resolution function was able to find a strategic (lateral) solution in all but two cases. In fact, out of 2307 conflicts generated in 572 simulated flight hours there were 2 instances where a resolution was not found. The case involved a 4-aircraft conflict Table 3: Impact of Traffic Density in which one of them lost separation with two of the intruders. Further analysis is required to identify the cause of the 2 resolution failures observed. As it was determined in earlier studies ${ }^{2}$, at traffic densities above 17 aircraft per 10,000 $\mathrm{nmi}^{2}$, the three resolution patterns used in computing strategic resolutions appear to provide insufficient maneuver flexibility in a few observed cases. It is hypothesized that the addition of maneuver patterns adds degrees of freedom and would provide the needed flexibility. The full implementation of AOP also uses a tactical resolution algorithm to handle exception cases in which a full strategic solution cannot be found before three minutes to go. This tactical capability was not exercised in this study. Also, adding a vertical degree of freedom to the resolution computation is likely to further improve the performance.

\section{B. Impact of Pilot Delay on LOS}

The effect of pilot delay with all pilots responding indicates a non-decreasing trend as both density and delay increase (Table 4). For that reason, it is important to perform an in-depth analysis of the results to identify the causes of the intrusions observed. Figure 5 depicts a notional timeline diagram of the conflict detection and resolution cycle in the presence of pilot delays from the viewpoint of the two aircraft involved. This diagram shows how, in some situations, it is possible that sufficient time may not be available to solve a conflict, thereby leading to an eventual LOS. In the diagram, Aircraft 1 and 2 are in conflict with each other. The conflict detection time horizon used in the study was 10 minutes, and therefore the alert for Aircraft 1 is shown with approximately 600 seconds remaining before LOS. The blue (solid) distribution indicates the range and probability of pilot delay, an input parameter for the simulation defined by a mean and standard deviation. The long mean delay condition of 240 seconds is shown. After some randomly selected pilot delay within this distribution, the pilot model requests a resolution and the resolution cycle begins. The green (dashed) distribution indicates the range of resolution cycle performance over a wide range of conflict geometries and traffic conditions. Depending on the specific traffic situation, a resolution trajectory may be easy (A) or hard (B) to compute, which results in an additional delay before the conflict is solved. If the situation is too complex, a resolution trajectory for Aircraft A may not be forthcoming. 
Meanwhile, the alert for Aircraft 2 is offset by three minutes to prevent synchronous conflict resolutions with Aircraft 1. Following this offset, the conflict alert is given and the pilot delay begins. As the figure shows, a three minute offset followed by a roughly four minute pilot delay leaves only about three minutes to solve the conflict. Strategic solutions may be more difficult to compute in this short time frame because maneuver options are limited; this can be seen by the notional difference in the resolution cycle performance distribution as compared to Aircraft 1. If the traffic situation is such that a solution is found (C), then the conflict is resolved (if not already resolved by Aircraft A). On the other hand, a strategic solution may be elusive in this time frame (D). In AOP, at one minute to LOS, the strategic CR algorithm is disabled. The current design for AOP is to switch to a tactical CR system at three minutes (180 seconds) to LOS. This tactical system was not tested in the current study. Therefore, at short time frames resulting from excessive pilot delays, the ability to solve conflicts strategically comes down to resolution cycle performance. Resolution cycle performance for a given algorithm design and implementation is generally a function of three factors: the traffic situation (including geometry and density), the time remaining before LOS, and the degrees of freedom available for generating possible solutions. Reductions of one or more of these parameters may have the effect of extending the time required

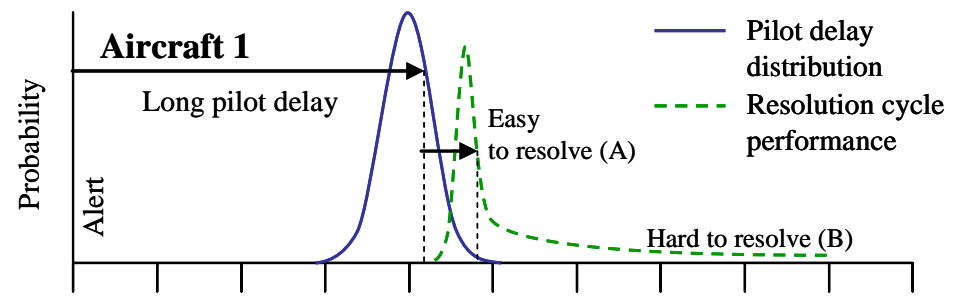
to find a conflict-free solution, and in

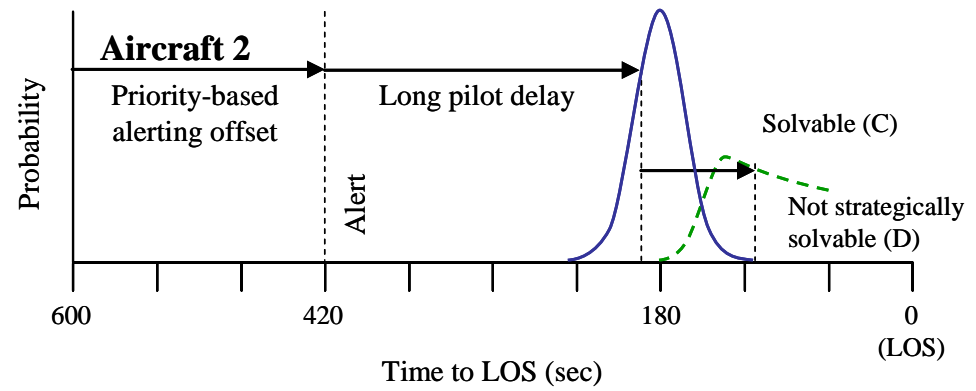

Figure 5: Conflict Detection and Resolution Timeline with Pilot Response Delay

some cases, may prevent such solutions altogether. Such cases would show up as LOS events provided that no other resolution system is present.

Of these factors in the current study, the traffic geometry was randomly generated, and the traffic density and pilot delay were parameters of the simulation. The remaining factor, degrees of freedom, was determined by the experiment setup and algorithm design. The experiment setup permitted no vertical solutions, which in itself significantly limited the degrees of freedom. In the lateral dimension, AOP searches three solution patterns: lateral offsets, path stretches, and direct intercepts ${ }^{20}$. These three patterns have been found to be largely adequate for solving randomly generated conflicts with full look-ahead time up to traffic densities of 17 aircraft per 10,000 $\mathrm{nmi}^{2}$. (See future work.) The detailed analysis on the causes of the observed LOS is fundamental to the design and optimization of CD\&R automation and it can only be done with high fidelity models. One of the conditions

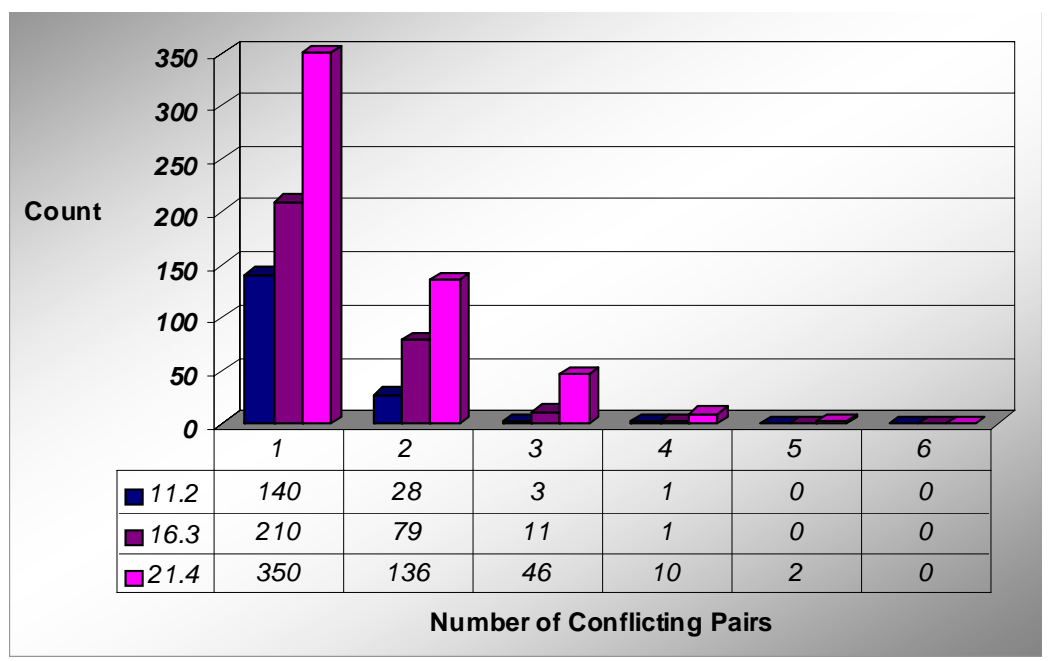

Figure 6: Number of multi-Aircraft Conflicts in a Single Run. that seems to affect the conflict resolution performance is the number of multi-aircraft conflicts encountered by the resolution logic. 
Figure 6 shows the distribution of multi-aircraft conflicts observed during three scenario runs corresponding to a pilot delay of 240 seconds. The graph shows the number of conflicts binned in terms of the number of conflicting aircraft pairs, for example three aircraft pairs indicate four aircraft involved (ownship plus three intruders). Note that the involved aircraft are not necessarily all in conflict with each other.

As Table 4 indicates, at the lowest traffic density of 11.2 aircraft per 10,000 $\mathrm{nmi}^{2}$, there was a single intrusion observed during the 240 seconds delay scenario. The intrusion was determined to be a multi-aircraft conflict. At the middle density, there were 5 LOS, 3 of them were minimal (distance at the point of closest approach was greater

Table 4: Experiment Data Runs per Density and Pilot Delay

\begin{tabular}{|c|c|c|c|c|c|c|}
\hline $\begin{array}{c}\text { Aircraft } \\
\text { per } \\
10000 \\
\mathrm{nmi}^{2}\end{array}$ & $\begin{array}{c}\text { Average } \\
\text { Pilot } \\
\text { Delay } \\
\text { (sec) }\end{array}$ & $\begin{array}{l}\text { Total } \\
\text { Flight } \\
\text { Hours }\end{array}$ & $\begin{array}{c}\text { Total } \\
\text { Conflicts }\end{array}$ & $\begin{array}{l}\text { Total } \\
\text { LOS }\end{array}$ & $\begin{array}{l}\text { LOS I } \\
\text { Conflicts }\end{array}$ & $\begin{array}{c}\text { LOS I Flight } \\
\text { Hour }\end{array}$ \\
\hline \multirow{4}{*}{$\begin{array}{c}11.2 \\
(5 X / 3 X)\end{array}$} & 3.5 & 240.73 & 583 & 0 & 0 & 0.000 \\
\hline & 46.0 & 59.21 & 150 & 0 & 0 & 0.000 \\
\hline & 90.0 & 300.38 & 744 & 0 & 0 & 0.000 \\
\hline & 240.0 & 59.52 & 142 & 1 & 0.0070 & 0.017 \\
\hline \multirow{4}{*}{$\begin{array}{c}16.3 \\
(8 X / 6 X)\end{array}$} & 3.5 & 90.71 & 316 & 1 & 0.0031 & 0.011 \\
\hline & 46.0 & 89.01 & 289 & 1 & 0.0034 & 0.011 \\
\hline & 90.0 & 87.03 & 284 & 1 & 0.0035 & 0.011 \\
\hline & 240.0 & 88.33 & 296 & 2 & 0.0067 & 0.023 \\
\hline \multirow{4}{*}{$\begin{array}{c}21.4 \\
(12 X / 8 X)\end{array}$} & 3.5 & 572.56 & 2307 & 2 & 0.0008 & 0.003 \\
\hline & 46.0 & 115.13 & 484 & 1 & 0.0020 & 0.009 \\
\hline & 90.0 & 577.48 & 2463 & 7 & 0.0028 & 0.012 \\
\hline & 240.0 & 117.35 & 514 & 10 & 0.0194 & 0.085 \\
\hline
\end{tabular}

than $4.92 \mathrm{nmi}$ ) the other two were multi-aircraft conflicts. At a density of 21.4 aircraft, there were ten LOS and nine of them involved multi-aircraft conflicts. The preceding analysis of the causes for the observed intrusions suggests that the complexity of the conflicts encountered and the delayed pilot response, both have an effect on the LOS metric.

\section{Impact of Delay and Density on the Resolution to LOS time}

The resolution execution time relative to the time of the predicted first loss of separation (FLOS) was found to be sensitive to pilot delay as expected. The detailed description of the cause for this response is provided in the preceding section, in Figure 4 and 5. This time is inversely related to pilot delay, reaching the lowest observed value in the experiment for pilot delays of 240 seconds as it can be seen in the graph in Fig. 7 . The effect of delayed resolutions on LOS begins to be observed in the experiment at 240 seconds. It is at that time that AOP strategic resolutions may not have time to converge to a solution prior to the 3minute-to-LOS limit. This metric shows

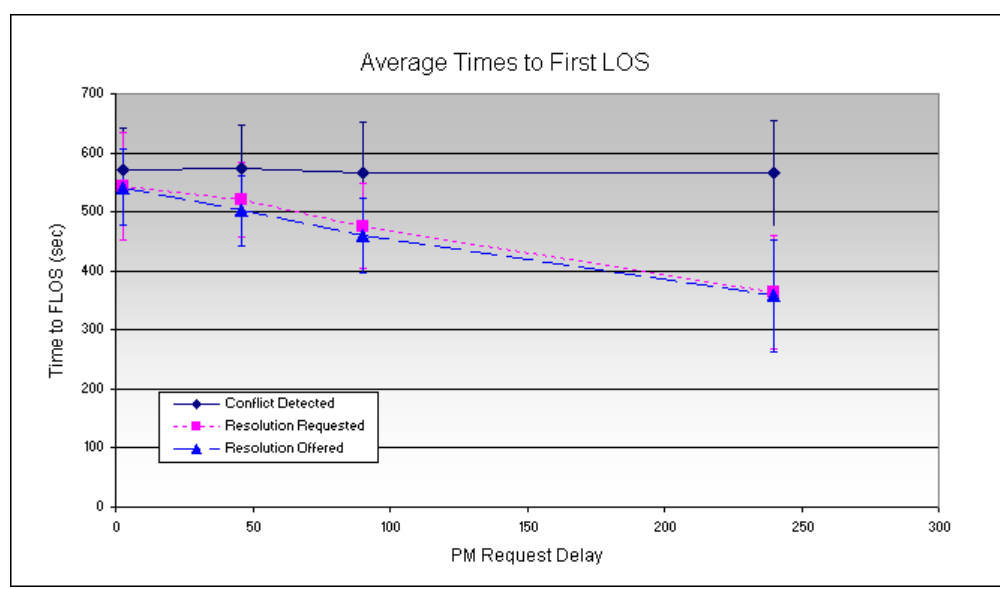

Figure 7: Average Time from Resolution to FLOS the resilience of the strategic CR to long pilot delays. 


\section{Impact of Pilot Responsiveness on LOS}

The effect of pilot responsiveness and traffic density for all combined pilot delays can be observed in Table 5 . When a pilot of one aircraft is non-responsive to a conflict alert, the onus is placed on the pilot of the other aircraft to resolve the conflict. As shown in Fig. 5, when one aircraft does not respond, the other aircraft still faces the same challenges in coming up with a strategic resolution in time. A significant delay caused either by the pilot or by the various factors that impact resolution cycle performance, discussed earlier, can result in no solution being found in sufficient time, therefore leading to a LOS. If the non-responsive pilot is flying Aircraft 1, then the priority-based alerting offset adds an additional delay which further increases the likelihood of a LOS. Of course, if both pilots are non-responsive, then a separation loss is assured. By multiplying the probability of non-responsiveness for any given aircraft (e.g., 10\%) by that of any aircraft it may be in conflict with (e.g., 10\%), the number of conflicts that result in a LOS due to this factor can be estimated (e.g. 1\% of conflicts). As Table 5 indicates, the percentage of LOS at the highest densities and lowest responsiveness levels is twice as much for the delays combined. (e.g., 2\%). In other words, the LOS shown are a combination of all the delay conditions.

Table 5: Data Runs shown by Pilot Responsiveness and Traffic Density

\begin{tabular}{|c|c|c|c|c|c|}
\hline $\begin{array}{c}\text { Non- } \\
\text { Resp. }\end{array}$ & Density & $\begin{array}{c}\text { Flight } \\
\text { Hours }\end{array}$ & $\begin{array}{c}\text { Total } \\
\text { Conflicts }\end{array}$ & $\begin{array}{c}\text { Total } \\
\text { LOS }\end{array}$ & $\begin{array}{c}\text { LOS I } \\
\text { Conflicts }\end{array}$ \\
\hline \multirow{3}{*}{0} & 11.2 & 659.84 & 1619 & 1 & 0.0006 \\
\cline { 2 - 6 } & 16.3 & 511.93 & 1711 & 7 & 0.0041 \\
\cline { 2 - 6 } & 21.4 & 1498.1 & 5799 & 37 & 0.0064 \\
\hline \multirow{4}{*}{98} & 11.2 & 0 & 0 & 0 & 0 \\
\cline { 2 - 6 } & 16.3 & 332.6 & 1121 & 7 & 0.0062 \\
\cline { 2 - 6 } & 21.4 & 457.15 & 1940 & 16 & 0.0082 \\
\hline \multirow{3}{*}{95} & 11.2 & 60.38 & 149 & 2 & 0.0134 \\
\cline { 2 - 6 } & 16.3 & 706.31 & 2297 & 18 & 0.0078 \\
\cline { 2 - 6 } & 21.4 & 350.77 & 1432 & 19 & 0.0133 \\
\hline \multirow{3}{*}{90} & 11.2 & 655.49 & 1505 & 41 & 0.0272 \\
\cline { 2 - 6 } & 16.3 & 266.39 & 887 & 10 & 0.0113 \\
\cline { 2 - 6 } & 21.4 & 1378.4 & 5652 & 114 & 0.0202 \\
\hline
\end{tabular}

\section{Conclusion and Future Work}

This study is part of an on-going series of high fidelity batch simulation experiments seeking to understand the safety impact of increased traffic density and system uncertainties. This paper studies the effects of human operator inattentiveness when interacting with cockpit based automated systems used for separation assistance. These effects were observed by varying pilot delays and responsiveness within wide ranges. LOS count and resolution to FLOS time were evaluated to assess the performance of the system under the experimental conditions. An in-depth analysis of the underlying causes for the observed behavior revealed great stability of the airborne strategic resolution capability under a large range of conditions. Results from this study can contribute to the design requirements for procedures and applications involving automated separation assurance systems. These results begin to reveal some of the traffic and system conditions that if not addressed could compromise safety such as operator response delay in very dense traffic. Finally, understanding the safety effects of human operator performance variability will enable the design of preventive procedures and more robust automation. Future experiments incorporating the tactical AOP capability as well as vertical resolution and possible optimized resolution patterns are being planned. Also, studies of other sources of uncertainties such as wind prediction errors and datalink communication degradation as well as mixed equipage operations are underway.

\section{Acknowledgments}

The research team acknowledges the valued assistance from Ed Scearce and the indispensable work by our ATOS simulation development team Doug Mielke, John Bunnell, Robert Vivona, David Karr, David Roscoe and Chris Wyatt. 


\section{References}

1. Joint Planning and Development Office: Concept of Operations for the Next Generation Air Transportation System.”, Version 2.0, June 2007. http://www.jpdo.gov/library/NextGen_v2.0.pdf

2. Consiglio, M., Hoadley, S., Wing, D., and Baxley, B. "Safety Performance of Airborne Separation: Preliminary Baseline Testing”, AIAA-2007-7739, 7th ATIO Conference, Belfast, Ireland, 2007

3. Sridhar, B., Sheth, K., Grabbe, S., “Airspace Complexity and its Application in Air Traffic Management”, $2^{\text {nd }}$ USA/Europe Air Traffic Management R\&D Seminar, Orlando, USA, December 1998

4. Laudeman, I.V., Shelden, S.G., Branstrom, R., and Brasil, C.L., "Dynamic Density: An Air Traffic Management Metric”, NASA-TM-1998-112226, April 1998.

5. Wing, D., “A Potentially Useful Role for Airborne Separation in 4D-Trajectory ATM Operations”, AIAA-20057336, ATIO, Washington DC, 2005

6. Chung, W. and Staab, R. "A 1090 Extended Squitter Automatic Dependent Surveillance - Broadcast (ADS-B) Reception Model for Air-Traffic-Management Simulations,” AIAA 2006-6614, Modeling and Simulation Technologies Conference and Exhibit, August 2006.

7. Karr, D., Roscoe, D., and Vivona R., "An Integrated Flight-Deck Decision-Support Tool in an Autonomous Flight Simulation,” AIAA Modeling and Simulation Technologies Conference and Exhibit, AIAA-2004-5261, August 2004

8. Karr, D., Roscoe, D., and Vivona R., "Conflict Detection Using Variable 4D Uncertainty Bounds to Control Missed Alerts,” AIAA Guidance, Navigation, and Control Conference and Exhibit, AIAA-2006-6057, August 2006

9. Karr, D., Roscoe, D., and Vivona R., "Conflict Detection Using Variable 4D Uncertainty Bounds to Control Missed Alerts,” AIAA Guidance, Navigation, and Control Conference and Exhibit, AIAA-2006-6057, August 2006

10. Bilimoria,K., Sheth, H., Grabble, S., "Performance Evaluation of Airborne Separation Assurance for Free Flight,” AIAA Guidance, Navigation, and Control Conference and Exhibit, AIAA 2000-4269, August 2000

11. Krozel, J., Peters, M., Bilimoria, K., Lee, C., and Mitchell, J., "Systems Performance Characteristics of Centralized and Decentralized Air traffic Separation Strategies,” 4th. USA/Europe Air Traffic Management R7D Seminar, Santa Fe, NM, December 2001.

12. Andrews, J., Erzberger, H., and Welch, J., "Safety Analysis for Advanced Separation Concepts," Air Traffic Control. Quarterly, Vol. 14, No. 1, 2006, pp. 5-24

13. Everdij, $\mathrm{M}$ and Blom, H., "Bias and Uncertainty Modeling in Accident Risk Assessment”, Distributed Control and Stochastic Analysis of Hybrid Systems Supporting Safety Critical Real-Time Systems Design (HYBRIDGE), February 2005. (http://hosted.nlr.nl/public/hosted-sites/hybridge/

14. Krystul, J. and Blom, H., "Monte Carlo Simulation of Rare Events in Hybrid Systems”, Distributed Control and Stochastic Analysis of Hybrid Systems Supporting Safety Critical Real-Time Systems Design (HYBRIDGE), July 2004. (http://hosted.nlr.nl/public/hosted-sites/hybridge/

15. Pritchett, A., Vandor, B., and Edwards, K., “Testing and Implementing Cockpit Alerting Systems”, Reliability Engineering and System Safety, V75-Issue 2, p193-206, 2002

16. Finkelstein, D., Lung, Vivona, R., Bunell, J., Mielke D., and Chung W.: “Airspace and Traffic Operations Simulation for Distributed ATM Research and Development.” AIAA 2005-6488.

17. FAA/Eurocontrol Cooperative R\&D Action Plan 1, "Principles of Operation for the Use of Airborne Separation Assurance Systems.”, Version 7.1, June $2001 . \quad$ http://www.eurocontrol.int/careasas/gallery/content/public/docs/po-asas71.pdf

18. RTCA Special Committee 186: “Application of Airborne Conflict Management: Detection, Prevention, \& Resolution.” RTCA/DO-263, December 2000.

19. Wing, D., Barmore, B., and Krishnamurthy, K., "Use of Traffic Intent Information by Autonomous Aircraft in Constrained Operations,” AIAA Guidance, Navigation, and Control Conference and Exhibit, AIAA-2002-4555, August 2002.

20. Vivona, R., Karr, D., and Roscoe, "Pattern-Based Genetic Algorithm for Airborne Conflict Resolution,” AIAA Guidance, Navigation, and Control Conference and Exhibit, AIAA-2006-6060, August 2006 